\title{
CAODV: Routing in Mobile Ad-hoc Cognitive Radio Networks
}

\author{
Angela Sara Cacciapuoti, Cosimo Calcagno, Marcello Caleffi \\ Department of Biomedical, Electronics and \\ Telecommunications Engineering \\ University of Naples Federico II \\ Naples, Italy \\ Email:\{angelasara.cacciapuoti, marcello.caleffi\}@unina.it, \\ cosimocalcagno@hotmail.com
}

\author{
Luigi Paura \\ Department of Biomedical, Electronics and \\ Telecommunications Engineering \\ University of Naples Federico II \\ Naples, Italy \\ Laboratorio Nazionale di Comunicazioni Multimediali \\ Naples, Italy \\ Email: paura@unina.it
}

\begin{abstract}
This paper deals with the routing in cognitive mobile ad hoc networks. We propose to modify the widely adopted Ad-hoc On-demand Distance Vector (AODV) protocol [1] in order to assure its functionality in the considered scenario. The resulting protocol, referred to as the Cognitive Ad-hoc Ondemand Distance Vector (CAODV) protocol, has been designed according to three guidelines: i) to avoid regions of primary users activity during both route formation and packet discovery without requiring a dedicated common control channel; ii) to perform a joint path and channel selection at each forwarder to minimize the route cost; iii) to take advantage of the availability of multiple channels to improve the overall performances. The performances of CAODV have been evaluated by means of numerical simulations, and the experimental results confirm its effectiveness for cognitive mobile ad hoc networks.

Index Terms - cognitive, ad hoc, manet, routing.
\end{abstract}

\section{INTRODUCTION}

The Cognitive Radio Networks (CRNs) paradigm has been recognized in 1999 [2] as an effective way to deal with bandwidth scarcity. Although almost ten years have passed, the research on CRNs has mainly focused [3] on MAC and physical issues, and few research has achieved in the area of routing for multi-hop CRNs.

In this paper, we consider a scenario in which mobile cognitive users (CUs) exploit multi-hop communications and primary user (PU) activity is dynamic, i.e. the primary spectrum band, once available, remains usable for a limited duration (in the order of minutes). In this scenario, the first priority is to build stable paths and, moreover, the path discovery process must be quick and resilient against PU activity changes.

As mentioned before, there is a limited amount of work available for such a scenario. The proposals in [4], [5], [6] require a dedicated common control channel and/or exploit a centralized approach, unfeasible in ad hoc scenarios. In [7] it is proposed a tree-based routing protocol for CRN but it requires infrastructured networks. In [8] another tree-based

This work is partially supported by the Italian National project "Global \& Reliable End to End e-Commerce \& On Line Service Platform" (GRECO) and by the Finanziamento per l'Avvio di Ricerche Originali (FARO) project "LATINO: Un sistema per la LocAlizzazione ed il Tracciamento di INdividui al fine di Ottimizzare percorsi in ambienti indoor". protocol has been proposed with reference to ad hoc networks, but assuming static or slowly moving CUs. Moreover, the proposed routing recovery mechanism requires that if a node is affected by a PU activity in a certain channel, then all the nodes (including those don't affected) must dismiss such a channel.

With reference to mobile scenarios, in [9] the authors propose a novel technique based on the probabilistic routing approach, but the problems of assigning node ids and of selecting efficient forwarding strategies have not been solved. Moreover, the latency for end-to-end communications can be very significantly large. In [10] a protocol based on the geographic routing paradigm has been proposed, assuming that the source and forwarders know the GPS position of the destination. Both the papers [11], [12] propose to enhance the AODV protocol for cognitive scenarios. The former utilizes a dedicated common control channel for broadcasting the route requests and replies. As a consequence, the data packets are routed along channels whose qualities have not been assessed. In [12] the authors do not resort to a dedicated channel but the assume that nodes are equipped with a single transceiver. However, the same issue about using un-assessed channels arises.

The proposed routing technique is based on a modification of the widely adopted Ad-hoc On-demand Distance Vector (AODV) protocol and, therefore, it will be referred to as Cognitive AODV (CAODV). Unlike most of the previous works, our proposal avoids regions of PU activity during both route formation and packet discovery without requiring any dedicated control channel. Moreover, it assesses the qualities of any available channel, minimizing the route cost by performing a joint path and channel selection at each forwarder. Finally, it exploits the presence of multiple available channels to improve the overall performances.

The rest of the paper is organized as follows. Section II describes the network architecture model and the related assumptions of the proposed approach, while in Section III we describe the CAODV protocol. A performance evaluation is provided in Section IV and, finally, Section V concludes the 
work.

\section{Network ARchitecture}

We assume that the network is composed by cognitive users (CUs) that freely move in a two-dimensional cartesian scenario. In the same scenario, the primary users (PUs), assumed to be stationary, operate according to a two-stage on/off switching cycle [10]. The number, the locations and the transmission standards of the PUs are assumed unknown to the CUs, and the PU activity is sensed by a spectrum sensing mechanism, which is out of the scope of this paper.

The CUs communicate through the licensed (primary) portion of the spectrum (i.e. there is no dedicated spectrum portion for $\mathrm{CU}$ communications) by means of $l$ channels, each having the same bandwidth. If a PU is active and its transmission frequency overlaps a CU channel, say channel $i$, this channel cannot be used by the CUs in the circular region centered at the PU position and with radius equals to the $\mathrm{PU}$ transmission range. Moreover, due to co-channel interference effects, the adjacent channels $i-2, i-1, i+1, i+2$ cannot be used as well in regions with a radius that decreases with the separation of the channels from channel $i$ as in [10].

Each CU is able to use the $l$ channels [10], perhaps at the same time. This assumption is reasonable if the CUs are equipped with multiple wireless interfaces. However, also in presence of a single wireless interface, assuming the presence of an underlying channel coordination mechanism [13], [14] the assumption holds.

\section{Cognitive Ad-hoc On-Demand Distance Vector}

In this section we provide an overview of the proposed protocol, by describing both the route discovery/maintenance process and the packet forwarding one and by highlighting the main differences with the original AODV protocol introduced for the implementation of the cognitive paradigm.

The route discovery process starts with a route request (RREQ) broadcasted by the source to neighbors on each channel not affected by a PU activity and ends with a route set up after the reception of a route reply (RREP) from the destination.

Similarly to AODV, the RREQs are broadcasted using an expanding ring search technique [1]. Differently from AODV, an intermediate $\mathrm{CU}$ is supposed to receive and handle RREQs and RREPs among a subset of the $l$ channels as shown by the flow charts depicted in Fig. 1 and 2. Moreover, unlike AODV, a node can store several routes, one for each available channel, and the routes can be composed by different intermediate nodes, i.e. they can refer to different paths. Finally, nodes must check for the presence of a PU before sending a packet through a channel.

More in detail, when an intermediate $\mathrm{CU}$ receives the first RREQ through a channel free from PU activity, say channel $i$, it sets up a reverse path toward the sender CU through the same channel. If the receiving $\mathrm{CU}$ can supply a valid route for the desired destination, then it sends a unicast route reply (RREP) back to the sender through the same channel. Otherwise, it

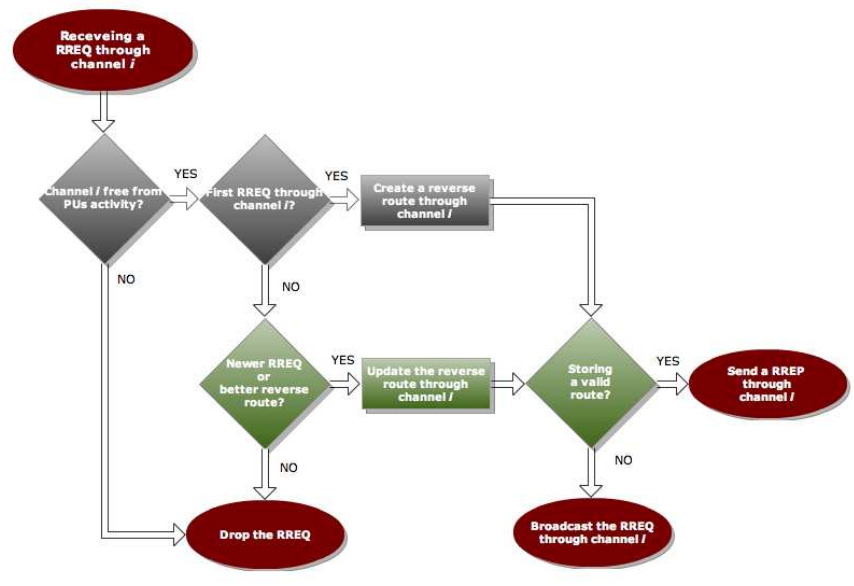

Fig. 1. RREQ packet flow chart

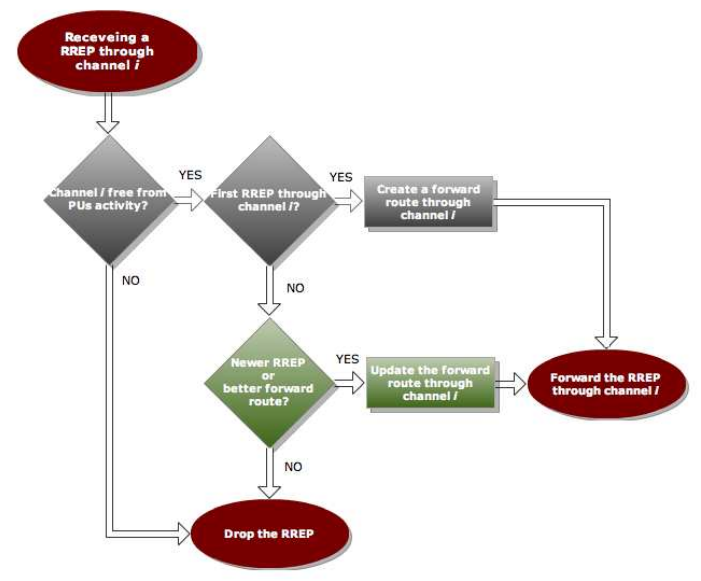

Fig. 2. RREP packet flow chart

broadcasts a copy of the RREQ packet through the channel $i$. If an additional RREQ is received through the same channel, the CU checks if the RREQ is newer or it refers to a better reverse route than the one stored in the routing table. In both cases the node updates the reverse path and it sends a RREP or it broadcasts the RREQ, differently the node simply discards the packet. We note that, since the route discovery processes associated with each channel are independent each other, it can happen that routes on different channels can be composed by different intermediate nodes, i.e. the CAODV exploits spatial diversity.

When an intermediate $\mathrm{CU}$ receives the first RREP through a free channel, say $i$, it sets up a forward route through the same channel toward the RREP sender and it forwards a copy of the RREP along the reverse path through channel $i$. If an additional RREP will be received through channel $i$, the CU will update the forward path only if the RREP is newer or it refers to a better forward route.

The route maintenance process aims to react to topology changes due to node mobility or wireless propagation instability as it occurs for AODV. However, differently from AODV, 


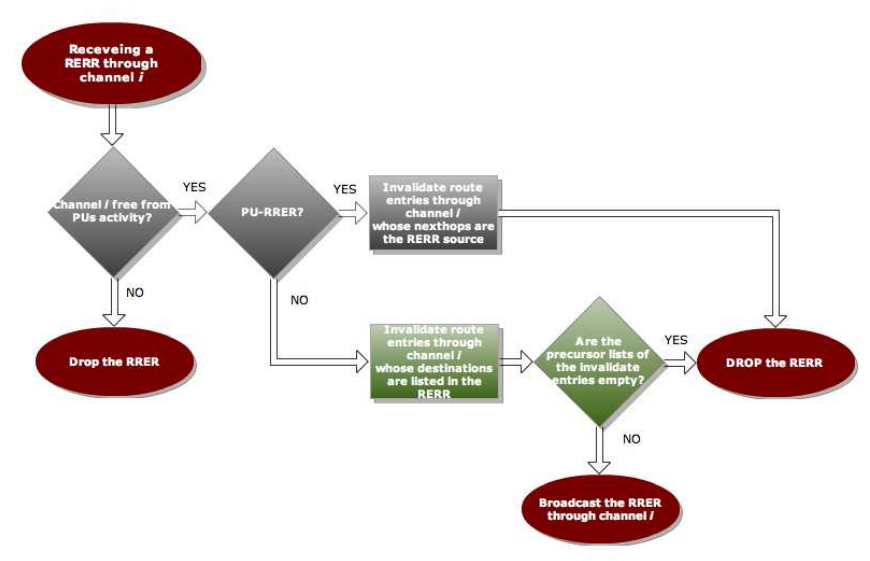

Fig. 3. RERR packet flow chart

a route error can be also due to a PU which starts to use a channel previously available. Therefore, CAODV exploits two classes of route error (RERR) messages: usual RERRs for handling topology changes and PU-RERRs for handling PU activity, as shown by the flow chart of Fig. 3.

When a PU activity is detected by a node in a channel, say $i$, the node invalidates all the routing entries through such a channel and it informs the neighbor CUs that channel $i$ is now unavailable with a PU-RERR packet. The CUs that receive such a packet invalidate the channel $i$ route entries whose next hop is the PU-RERR source. In such a way, only the nodes effectively affected by the PU activity must release the busy channel. We note also that, unlike usual RERRs, the scope of the PU-RERR packets is local, since they are never reforwarded and they do not rise to new route requests. This allows the CAODV to limit the overhead in case of frequent changes in the activity of the PUs. As a consequence, it can happen that a data packet reaches an intermediate $\mathrm{CU}$ whose entry has been invalidated with a PU-RERR, and in such a case a usual RERR is broadcasted by the intermediate CU.

On the other hand, when a node senses that a previously occupied channel becomes available, it re-validate the stored routing entries through channel $i$ so that incoming data packets may be forwarded along such a channel. We underline that no control packets are sent in such a case.

As regards to the packet forwarding process, in order to maximize the spectrum efficiency, CAODV exploits all the available channels to improve the overall performances. To this aim, each forwarder, at the first, singles out the shortest available paths for the destination, then, it selects by chance one of the shortest paths and forwards the data packet through it. In such a way, CAODV not only benefits from spectral diversity increasing the packet delivery ratio, but it also keeps active all the shortest routes.

\section{Performance Evaluation}

In this section we validate the CAODV protocol with numerical simulations via Network Simulator 2 (ns-2) [15] under different environments, network conditions and PU activities. The ns-2 has been extended to multi-radio multichannel environments according to [16]. Unfortunately, since no one routing protocol for ad hoc CRNs has been publicly released as source code, we cannot assess a performance comparison with other protocols. However, we set the simulation scenarios as close as possible to those adopted in [10] so that a qualitative comparison can be stated. Moreover, a fair comparison with AODV has been carried out to validate the proposed implementation.

More in detail, CUs move according to the random waypoint model in a square area, whose size has been set such as it fits with a node density equal to 400 nodes $/ K \mathrm{~m}^{2}$. The transmission range of the CUs has been set to 120 meters, the transmission standard is the IEEE $802.11 \mathrm{~b}$ for each of the 10 channels and the propagation model is the Two-Ray Ground one. The transmission range of the PUs, assumed stationary, has been set to 300 meters and their activity is modeled according to a two stage ON/OFF process with exponential distribution with parameter $\lambda$, referred in the following as PU activity time. The workload is modeled as CBR data packets 1000 bytes long over UDP connections, and each node generates one data flow toward a destination selected by chance. To effectively assess the scalability property of the analyzed protocol, according to the Gupta-Kumar [17] bound we set the data throughput generated by each source to $\frac{W}{10 \sqrt{n}}$, where $W$ is the link data throughput for a $802.11 \mathrm{~b}$ channel with CCK11 modulation (about $5.4 \mathrm{Mb} / \mathrm{s}$ ) and $n$ is the number of CUs in the network.

The duration of each run is 1060 seconds, the data traffic is active in the interval $[60,1000]$ seconds, and for each experiment we performed five runs computing both the average value and the standard deviation for each metric, that is: i) delivery ratio: the ratio between the number of data packets successfully received and those generated; ii) hop count: the number of hops for a data packet to reach its destination (this metric accounts only for the data packets successfully received); iii) routing overhead: the ratio between the number of generated data packets and the total number of generated routing packets.

In the first experiment (Fig. 4-6) we validate the proposed protocol by comparing its performances with those of AODV as the number of nodes in the network increases. For a fair comparison, the number of available channels has been set to one and no PUs are present in the scenario. We observe

TABLE I

SIMULATION PARAMETERS

\begin{tabular}{ll}
\hline CU number & {$[20, \ldots, 100]$} \\
CU transmission range & $120 \mathrm{~m}$ \\
CU node density & $400 \mathrm{nodes} / \mathrm{Km}^{2}$ \\
maximum CU speed & $2 \mathrm{~m} / \mathrm{s}$ \\
PU number & {$[2, \ldots, 18]$} \\
PU tx range for the overlapped channel $i$ & $300 \mathrm{~m}$ \\
PU tx range for adjacent channels $(i-1, i+1)$ & $150 \mathrm{~m}$ \\
PU tx range for adjacent channels $(i-2, i+2)$ & $75 \mathrm{~m}$ \\
PU activity parameter $\lambda$ & {$[50, \ldots, 400]$} \\
\hline
\end{tabular}




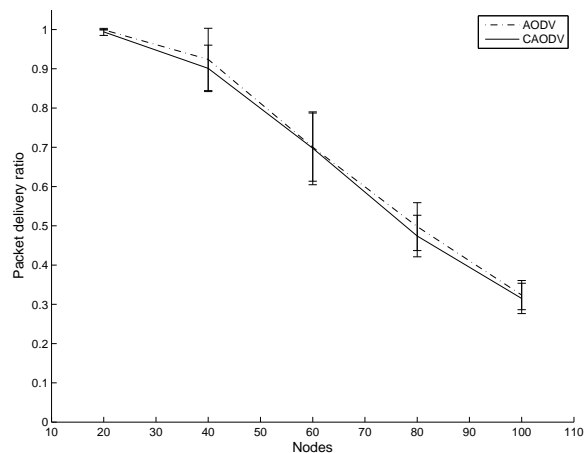

Fig. 4. PDR vs Nodes number

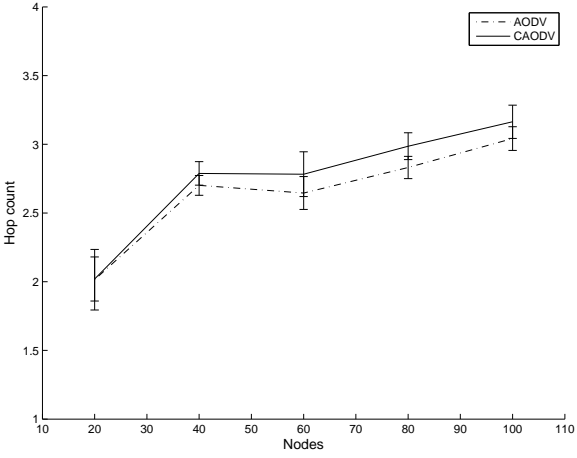

Fig. 5. Hop count vs Nodes number

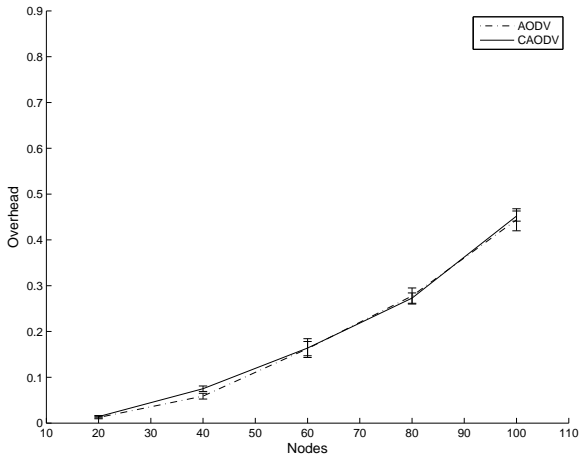

Fig. 6. Overhead vs Nodes number that there is a good agreement between the AODV and the CAODV performances, and the negligible differences are due to changes carried on to the ns- 2 structure to account for multiradio multi-channel environments. Moreover, we note that the PDR decreasing as the node number increases is due to the congestion caused by the data traffic, which scales with $\sqrt{n}$.

In the second experiment (Fig. 7-9), we evaluate the CAODV performances as the number of CUs $n$ increases. We set the PU number to 10 and the PU activity parameter $\lambda=200$ s. With reference to the PDR shown in Fig. 7, we observe that when the $\mathrm{CU}$ number is low, i.e. $n=20$ or $n=40$, the PDR is low as well, while for higher values the performances increase, reaching almost $90 \%$ of delivered packets when $n=100$. This behavior is reasonable and confirmed by the results in terms of hop number shown in Fig. 8. For low values of $n$, i.e. for small areas (fixed node density), each node is affected by the activity of all the PUs and, hence, it is often isolated due to the unavailability of free channels. Therefore, the packets delivered are mainly those sent when most of the PUs are inactive and directed to destinations very close to the sources, as confirmed by the average value of hop count metric, roughly 2 . On the other hand, when $n$ increases the area increases as well and, therefore, CAODV is able to build paths unaffected by PUs activity for most of the flows. Also the results in terms of routing overhead agrees with the previous comment: for low values of $n$, the unavailability of free channels inhibits the nodes from starting route requests, limiting so the routing overhead.

In the third experiment (Fig. 10-12), we analyze the CAODV performances as the number of PUs $(m)$ increases. We set the CU number to 50 and the PU activity parameter $\lambda=200$ s. As expected, we observe that the performances in terms of PDR decreases with the number of PUs, decreasing the number as well as the duration of the free channels. The hop count metric is almost unaffected by the PU number, while on the contrary the routing overhead is largely affected by the PU number increasing.

In the last experiment, we evaluate the CAODV performances as the PU activity time increases, namely as $\lambda$ increases. The results prove that, for the considered values,
CAODV is robust with respect to the PU activity time, building so stable paths.

\section{CONCLUSiON}

In this paper a routing protocol operating in mobile ad hoc cognitive radio networks has been proposed. The protocol, referred to as Cognitive Ad-hoc On-demand Distance Vector, exhibits three key features: i) unlike most of the previous works, it avoids regions of primary users activity without requiring any dedicated control channel; ii) it assesses the quality of any available channel by means of RREQ and RREP packets and it minimizes the route cost by performing a joint path and channel selection at each forwarder; iii) it exploits the presence of multiple available channels to improve the overall performances. Numerical simulations validate the proposed protocol, although an extensive performance analysis must be carried on to recognize its effectiveness in different operating conditions. Moreover, more effective route metrics can be considered.

\section{REFERENCES}

[1] C. E. Perkins and E. M. Royer, "Ad hoc on-demand distance vector (aodv) routing," Internet Engineering Task Force (IETF), Request for Comments 3561, July 2003.

[2] I. Mitola, J. and J. Maguire, G.Q., "Cognitive radio: making software radios more personal," Personal Communications, IEEE, vol. 6, no. 4, pp. $13-18$, aug 1999.

[3] H. Khalifé, N. Malouch, and S. Fdida, "Multihop cognitive radio networks: to route or not to route," IEEE Network, vol. 23, no. 4, pp. 20-25, 2009.

[4] C. Xin, B. Xie, and C.-C. Shen, "A novel layered graph model for topology formation and routing in dynamic spectrum access networks," in New Frontiers in Dynamic Spectrum Access Networks, 2005. DySPAN 2005. 2005 First IEEE International Symposium on, November 2005, pp. $308-317$.

[5] R. Pal, "Efficient routing algorithms for multi-channel dynamic spectrum access networks," in New Frontiers in Dynamic Spectrum Access Networks, 2007. DySPAN 2007. 2nd IEEE International Symposium on, April 2007, pp. $288-291$.

[6] G. Cheng, W. Liu, Y. Li, and W. Cheng, "Joint on-demand routing and spectrum assignment in cognitive radio networks," in Communications, 2007. ICC '07. IEEE International Conference on, 24-28 2007, pp. 6499 -6503 .

[7] B. Zhang, Y. Takizawa, A. Hasagawa, A. Yamaguchi, and S. Obana, "Tree-based routing protocol for cognitive wireless access networks," in Wireless Communications and Networking Conference, 2007.WCNC 2007. IEEE, 2007, pp. $4204-4208$. 


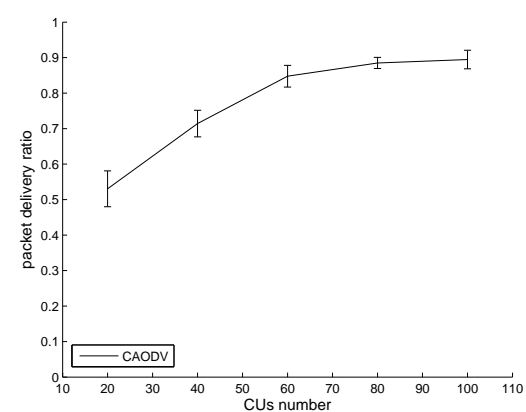

Fig. 7. PDR vs CU number

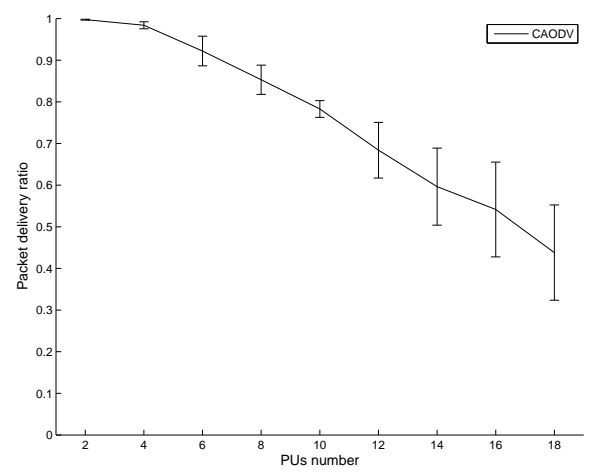

Fig. 10. PDR vs PU number

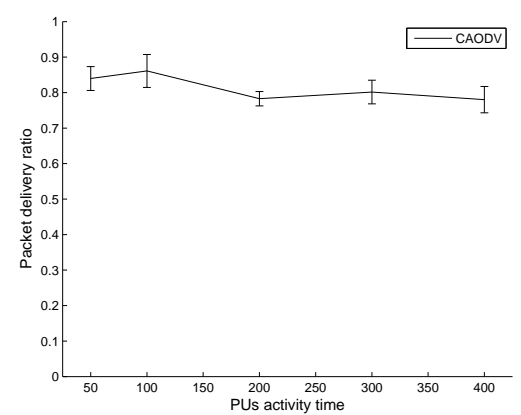

Fig. 13. PDR vs PU activity time

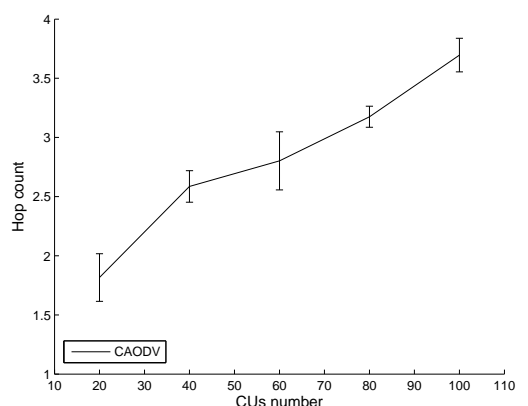

Fig. 8. Hop count vs CU number

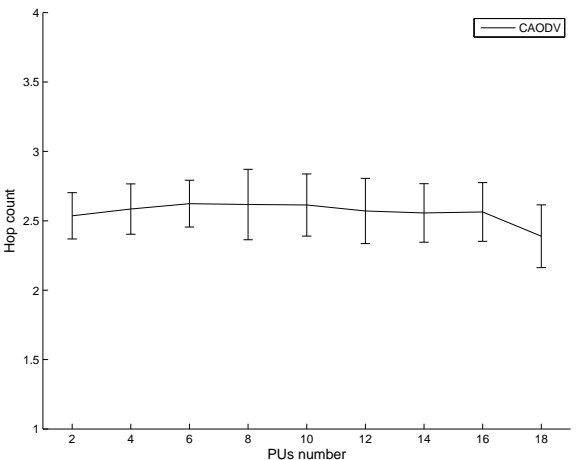

Fig. 11. Hop count vs PU number

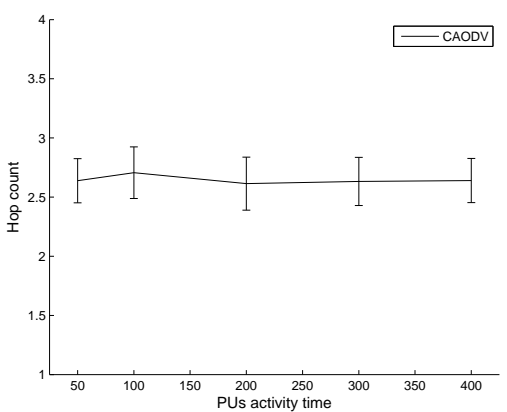

Fig. 14. Hop count vs PU activity time

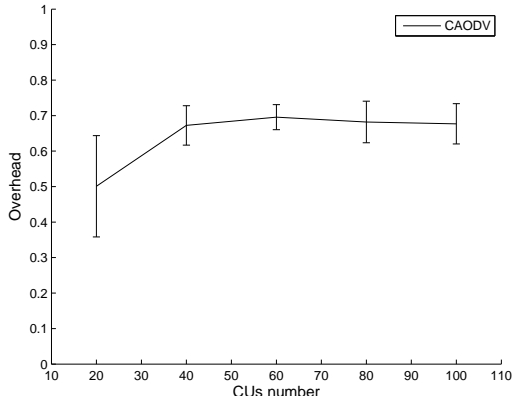

Fig. 9. Overhead vs CU number

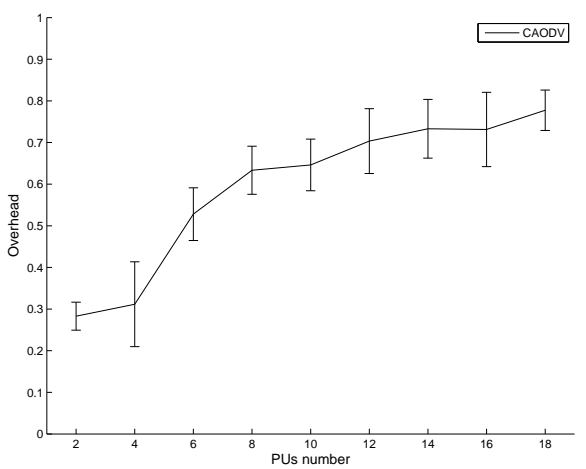

Fig. 12. Overhead vs PU number

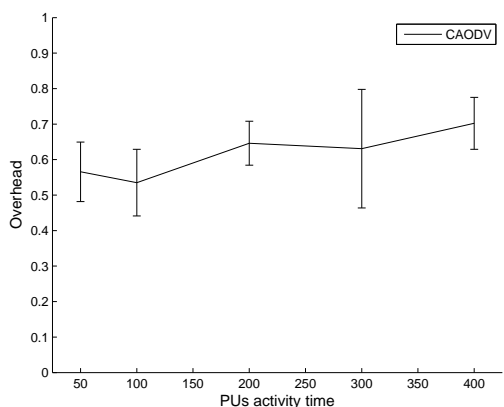

Fig. 15. Overhead vs PU activity time
[8] G.-M. Zhu, I. Akyildiz, and G.-S. Kuo, "Stod-rp: A spectrum-tree based on-demand routing protocol for multi-hop cognitive radio networks," in Global Telecommunications Conference, 2008. IEEE GLOBECOM 2008. IEEE, nov. 2008, pp. $1-5$

[9] A. C. Talay and D. T. Altilar, "Racon: a routing protocol for mobile cognitive radio networks," in CoRoNet '09: Proceedings of the 2009 ACM workshop on Cognitive radio networks, 2009, pp. 73-78.

[10] K. R. Chowdhury and M. D. Felice, "Search: A routing protocol for mobile cognitive radio ad-hoc networks," Computer Communications, vol. 32, no. 18, pp. 1983-1997, 2009.

[11] G. Cheng, W. Liu, Y. Li, and W. Cheng, "Spectrum aware on-demand routing in cognitive radio networks," in New Frontiers in Dynamic Spectrum Access Networks, 2007. DySPAN 2007. 2nd IEEE International Symposium on, April 2007, pp. $571-574$.

[12] H. Ma, L. Zheng, X. Ma, and Y. luo, "Spectrum aware routing for multi-hop cognitive radio networks with a single transceiver," in $\operatorname{Cog}$ - nitive Radio Oriented Wireless Networks and Communications, 2008. CrownCom 2008. 3rd International Conference on, May 2008, pp. 1 $-6$.

[13] J. So and N. H. Vaidya, "Multi-channel mac for ad hoc networks: handling multi-channel hidden terminals using a single transceiver," in MobiHoc '04: Proceedings of the 5th ACM international symposium on Mobile ad hoc networking and computing, 2004, pp. 222-233.

[14] C. Cormio and K. R. Chowdhury, "A survey on mac protocols for cognitive radio networks," Ad Hoc Networks, vol. 7, no. 7, pp. 1315 - 1329, 2009.

[15] T. V. Project, "The ns manual (formerly ns notes and documentation)."

[16] R. A. Calvo and J. P. Campo, "Adding multiple interface support in ns-2." [Online]. Available: http://personales.unican.es/aguerocr/files/ ucMultilfacesSupport.pdf

[17] P. Gupta and P. Kumar, "The capacity of wireless networks," Information Theory, IEEE Transactions on, vol. 46, no. 2, pp. 388-404, Mar 2000. 